\title{
Influence of malaria infection on peroxyl-radical trapping capacity in plasma from rural and urban Thai adults
}

\author{
BY D. I. THURNHAM*, RATREE SINGKAMANI $\dagger$ R. KAEWICHIT† AND \\ KALAYA WONGWORAPAT $\dagger$
}

Nutrition Studies Group, Clinical Investigation Unit, Dudley Road Hospital, Birmingham B18 7QH

(Received 7 July 1989 - Accepted 5 February 1990)

\begin{abstract}
Measurement of peroxyl-radical trapping capacity (TRAP) were made in plasma from patients with malaria from a rural and an urban Thai community. The results were compared with those from control subjects living in the same areas and chosen to match the patients closely. Measurements were also made of various antioxidants including nutritional indices vitamin $C$ and $\alpha$-tocopherol and the non-nutritional indices urate and protein-sulphydryl. Parasite counts, temperature on examination and the duration of illness were recorded together with measurements of plasma caeruloplasmin (EC 1.16.3.1), retinol and malondialdehyde (MDA). In general, most measurements made in the villagers were lower than those in the comparable urban groups. The exceptions were caeruloplasmin and MDA when the latter was expressed as MDA : cholesterol ratio. TRAP values were extremely low in $50 \%$ of the villagers and $25 \%$ of the urban patients with malaria and these results correlated with retinol and vitamin $C$ and inversely with malonaldehyde. The results suggested that low TRAP values are associated with lipid peroxidation and that vitamin $C$ and possibly retinol may be destroyed by the oxidative conditions present in the plasma in this disease.
\end{abstract}

Antioxidants: Malaria : Radical trapping capacity

Lipid peroxidation is believed to be intimately involved in the aetiology of a wide range of diseases including cancer (Slater, 1984), rheumatoid arthritis (Blake et al. 1989), coronary heart disease (Gey, 1986), alcohol abuse (DiLuzio \& Kalish, 1966) and the degenerative changes associated with ageing (Harman, 1984). Malaria was added to this list by Clark et al. (1976) who showed that antibody-independent immune mechanisms play a significant role in protecting the host against acute malarial infections and that mononuclear phagocytes were probably involved in this process. Highly reactive oxygen species (ROS) produced by these phagocytic cells have been shown to kill intra-erythrocytic parasites under both in vivo and in vitro experimental conditions (Clark \& Hunt 1983; Wozencraft et al. 1984), but it has been suggested that some aspects of severe malaria may result from the exaggerated effects of ROS on host tissues (Clark et al. 1986; Clark, 1987).

To prevent oxidative damage, tissues contain widely distributed enzymes to detoxify those intermediates that initiate lipid peroxidation, i.e. superoxide dismutase (EC 1.15.1.1), glutathione peroxidase $(E C 1.11 .1 .9)$ and catalase $(E C 1.11 .1 .6)$. However, if peroxidation does take place, there are also a number of substances present in the body that sequester lipid-free radical intermediates of the autocatalytic chain reaction. These chainbreaking substances include several dietary nutrients: $\alpha$-tocopherol, ascorbate, carotenes, etc., and it seems probable that dietary deficiencies of these nutrients will impair the body's protection against lipid peroxidation.

Present addresses: *MRC Dunn Nutrition Laboratories, Downhams Lane, Milton Road, Cambridge CB4 IXJ. $\dagger$ Research Institute of Health Sciences, PO Box 80 CMU, Chiang Mai 50002, Thailand. 
Plasma is in close contact with all the elements of the host-pathogen interaction in malaria. To examine the influence of this interaction on the host's oxidant defence system, we recently measured the capacity of plasma from patients with malaria to trap peroxyl radicals (Thurnham et al. 1988a). This method, known as the TRAP assay, was described by Wayner et al. $(1985,1987)$ and measures the combined radical-trapping capacity of $\alpha$ tocopherol, ascorbate, urate and protein-sulphydryl groups. Measurements of TRAP in plasma from malaria-infected Nigerian children showed very low to undetectable capacity to block peroxyl radical generation. However, similar results were obtained in children with no malaria but who had other infections, typically upper respiratory chest infections and varying degrees of malnutrition, and no factor emerged as being specifically linked to the low TRAP values.

The present study was set up to examine plasma from malaria patients with different social and nutritional backgrounds. The patients were mainly adults, so the likelihood of other concurrent infections in either patients or controls was less likely than with children. The results suggested that nutritional status may interact with malaria to depress TRAP values. Brief reports of some of these findings have appeared elsewhere (Singkamani et al. 1988; Thurnham, 1988).

\section{EXPERIMENTAL}

\section{Subjects}

Blood was taken over 2 weeks from twenty villagers (eleven men, eight women and one male child, age range 6-38 years) who presented with malaria at Maejam District Hospital, an area approximately $120 \mathrm{~km}$ South-east of Chiang Mai in Northern Thailand. All patients were from farming families and were infected with Plasmodium falciparum. Malaria was diagnosed on the basis of clinical symptoms and a parasite-positive blood film. Thick blood films were made in the out-patients' clinic and stained with Giemsa. Unfortunately some of the slides were damaged before accurate counts were made and cannot be reported. Blood was taken from sixteen control subjects who were relatives accompanying the patients to hospital: there were eight men, seven women and one male child, age range 7-34 years. In all cases blood samples were taken at the clinic where the patients attended for treatment. Details on duration of fever were obtained by enquiry.

The second group of malaria patients were twenty men and four women, age range 17-49 years, who presented at the out-patient clinic of the Department of Communicable Diseases, Malaria Control Region 2, Ministry of Public Health, Chiang Mai. This group were predominantly town-dwellers with many different occupations (Government officer, soldier, monks, lorry driver, tour guide, merchants, housewife, manual workers and a hairdresser). Malaria was identified as $P$. vivax in twelve cases, $P$. falciparum in ten cases, there was one with a mixed infection and one that could not be identified. Controls for these patients were recruited from university and hospital staff in Chiang Mai (thirteen men, $26-45$ years). Control subjects included gardeners, drivers, auxilliary and laboratory workers with salaries covering the same range as the patients.

We matched the sex ratios and age-ranges of controls against patients as closely as possible for the respective rural and urban groups. Unfortunately, several samples could not be used in the study as the blood was haemolysed, hence the uneven numbers described previously. A few of these additional samples were included for vitamin $\mathrm{C}$ measurements of the rural groups, as the samples were stabilized at the time the blood was collected. All control subjects were apparently healthy and, therefore, blood films were not examined for malaria. Ethical approval for these studies was obtained from the Health Sciences Ethical Committee, Chiang Mai University. 


\section{Biochemical methods}

Blood was collected in sequestrine tubes in both places. After centrifuging, plasma was stored at $-20^{\circ}$ for $2-3 \mathrm{~d}$ (Chiang Mai samples) or 2 weeks (village samples). They were then transported in ice to $\mathrm{a}-70^{\circ}$ freezer and stored for a further 4 weeks. Finally they were brought to England in dry ice, kept at $-45^{\circ}$ before analysis and all TRAP measurements were made within 4 weeks.

\section{Total radical trapping parameter (TRAP)}

The TRAP assay is based on that described by Wayner et al. $(1985,1987)$ and we have made only minor modifications (Thurnham et al. 1988a). Reagents were obtained from BDH (Poole, Dorset) unles otherwise indicated. The system comprises a water-soluble, thermolabile, free-radical initiator 2,2'-azo-bis-(2-amidinopropane hydrochloride) (ABAP; Polysciences, Warrington, PA, USA) in phosphate-buffered saline (PBS; $9 \mathrm{~g}$ sodium chloride/1, pH 8) which peroxidizes linoleic acid in a Clarke-type $\mathrm{O}_{2}$ electrode (Model 5331; Yellow Springs Instrument Co., Yellow Springs, OH, USA) and is measured by monitoring $\mathrm{O}_{2}$ uptake. In the routine assay, $250 \mu \mathrm{l}$ serum or plasma were vortexed for $3045 \mathrm{~s}$ with $10 \mu \mathrm{l}$ linoleic acid (LA; Sigma, Poole, Dorset; concentration of added LA in plasma approximately $137 \mathrm{mmol} / \mathrm{l}$ ). Of the mixture $50 \mu \mathrm{l}$ were added to $3 \mathrm{ml}$ air-saturated PBS in the $\mathrm{O}_{2}$ electrode chamber and the reaction was started by addition of $30 \mu \mathrm{l} 0.4 \mathrm{~mol} \mathrm{ABAP} / \mathrm{l}$. $\mathrm{O}_{2}$ uptake was monitored for approximately $40 \mathrm{~min}$ until the rate of uptake reached its maximum. When approximately $50 \%$ of the $\mathrm{O}_{2}$ in the chamber had been utilized, $10 \mathrm{nmol}$ 6-hydroxy-2,5,7,8-tetramethylchroman-2-carboxylic acid $(25 \mu 1 \quad 0.4 \mathrm{mmol} / 1$, Trolox; Hoffmann-La Roche, Nutley, NJ, USA) were added to calibrate the system. Addition of serum and Trolox retarded $\mathrm{O}_{2}$ uptake (induction times) until all antioxidant was consumed in quenching peroxyl radicals, and induction times were measured from the recorder trace. The total assay time for a normal serum sample was approximately $90 \mathrm{~min}$. TRAP is defined as $\mu \mathrm{mol}$ peroxyl radicals trapped/1 serum or plasma and was calculated from the following equation:

$$
\operatorname{TRAP}(\mu \mathrm{mol} / 1)=n[\text { Trolox }] \times(1000 / 50) \times(T \text { serum } / T \text { Trolox }),
$$

where $n$ is the stoichiometric factor for Trolox, which equals 2 (Wayner et al. 1985, 1987); $T$ is induction time, i.e. time taken to achieve maximum $\mathrm{O}_{2}$ uptake following addition of sample or Trolox and measured from the recorder trace; [Trolox] is $10 \mathrm{nmol}$ added in $25 \mu 1$.

\section{Stoichiometric factors}

In the present study we have used stoichiometric factors of $2,1.5,1.7$ and 0.2 for $\alpha$ tocopherol, ascorbate, urate and protein-sulphydryl respectively (Koottathep, 1988) to calculate theoretical TRAP values from the measured concentrations of the individual components.

\section{Fat-soluble vitamins}

Tocopherol, retinol and carotenoids were measured using high-performance liquid chromatography (Thurnham et al. $1988 \mathrm{~b}$ ). Plasma $(0.25 \mathrm{ml}$ ) was mixed with $0.25 \mathrm{ml}$ sodium dodecyl sulphate $(10 \mathrm{mmol} / 1)$ and $0.5 \mathrm{ml}$ ethanol containing internal standard (tocopherol acetate), and extracted into $1.0 \mathrm{ml}$ heptane. After evaporation and reconstitution in $0.25 \mathrm{ml}$ mobile phase (acetonitrile-methanol-chloroform, 47:47:6, by vol.), $25 \mu 1$ were injected onto a $60 \mathrm{~mm}, 3 \mu \mathrm{m}$ ODS- 2 cartridge chromatography column (Pharmacia) and separated at $0.5 \mathrm{ml} / \mathrm{min}$. Tocopherol, retinol and carotenes were monitored at 292, 325 and $450 \mathrm{~nm}$ respectively using a Millipore-Waters model 490 detector. The readings were quantified using appropriate extinction coefficients with standards obtained from Sigma 
(Poole, Dorset). Minimum detection level for retinol and $\alpha$-tocopherol was $0.01 \mu \mathrm{mol} / \mathrm{l}$ and for the carotenes $0.001 \mu \mathrm{mol} / \mathrm{l}$. Coefficients of variation varied from 5 to $10 \%$ (Thurnham et al. 1988b).

\section{Urate}

Urate was measured in metaphosphoric acid extracts of $0 \cdot 1 \mathrm{ml}$ plasma (Wayner et al. 1985) using a modified procedure set up to measure dehydroascorbate (Thurnham et al. 1988a). An internal standard (3,4-dihydroxybenzylamine hydrobromide; Aldrich Chemicals, Milwaukee, USA) was added during the extraction and $50 \mu \mathrm{l}$ samples were injected onto a $250 \times 4.6 \mathrm{~mm}$ column of $10 \mu \mathrm{m}$ particles of Lichrosorb- $\mathrm{NH}_{2}$ (Technicol Ltd, Stockport, Cheshire) and eluted at $2.0 \mathrm{ml} / \mathrm{min}$ with acetonitrile-sodium dihydrogen phosphate $(25 \mathrm{mmol} / \mathrm{l})(\mathrm{pH} \mathrm{4.8})$ mixed in the ratio 78.6:21.4 (v/v). Absorbance of urate was measured at $254 \mathrm{~nm}$ and 0.01 absorption units full scale (Spectromonitor III; LDC Ltd, Stone, Staffordshire).

\section{Other analyses}

Protein-sulphydryl. Protein-sulphydryl concentrations were measured usng DTNB reagent at $\mathrm{pH} 7 \cdot 4$ (Koster et al. 1986).

Malondialdehyde (MDA). MDA was measured in $0.2 \mathrm{ml}$ plasma (Satoh, 1978) using 1,1,3,3-tetraethoxypropane (Sigma) as standard.

Caeruloplasmin. Caeruloplasmin was measured using Nor-Partigen immunodiffusion plates and appropriate protein standard (Behring-Hoechst UK Ltd, Hounslow, Middlesex).

Cholesterol. Plasma cholesterol was measured enzymically (kit 14138/40; Merck Ltd, Poole, Dorset).

Total ascorbate. Plasma $(0.2 \mathrm{ml})$ from twenty rural patients (twelve men, eight women) and twenty rural control subjects (twelve men, eight women) was stabilized with $0.8 \mathrm{ml}$ trichloroacetic acid $(50 \mathrm{~g} / 1)$ at the time of blood collection. The extracts were transported to the UK with the samples and measured colorimetrically following reaction with 2,4dinitrophenylhydrazine (Thurnham \& Stephen, 1979).

\section{Statistical methods}

Non-parametric methods have been used to compare groups unless otherwise stated as the data tended not to be normally distributed.

\section{RESULTS}

The distribution of TRAP measurements in the four groups of subjects is shown in Fig. 1 and the median and ranges in Table 1. Median TRAP capacity was significantly lower in the rural malaria patients than all other groups and in this group 50\% (ten of twenty) had TRAP measurements less than $100 \mu \mathrm{mol} / 1$. In the urban malaria patients, although the median TRAP value was no different from the other groups, $23 \%$ (five of twenty-two) of these patients had similar low values. There was only one person in the two control groups with such a low TRAP value and he was subsequently found to have an abnormal haemoglobin disease.

In contrast, if the TRAP capacity was calculated from the individual concentrations of $\alpha$-tocopherol, urate and protein-sulphydryl (TRAP ${ }^{\text {calc }}$ ), median TRAP ${ }^{\text {cale }}$ values of the different groups (Table 1) were very similar and only the median TRAP ${ }^{\text {cale }}$ result from the urban control group differed significantly from the other three. Within the different groups, there was no difference between TRAP ${ }^{\text {calc }}$ and experimentally determined TRAP values except in the group of rural malaria patients $(P<0.002$, paired $t$ test $)$.

For the individual radical trapping antioxidants, i.e., $\alpha$-tocopherol, urate and protein- 


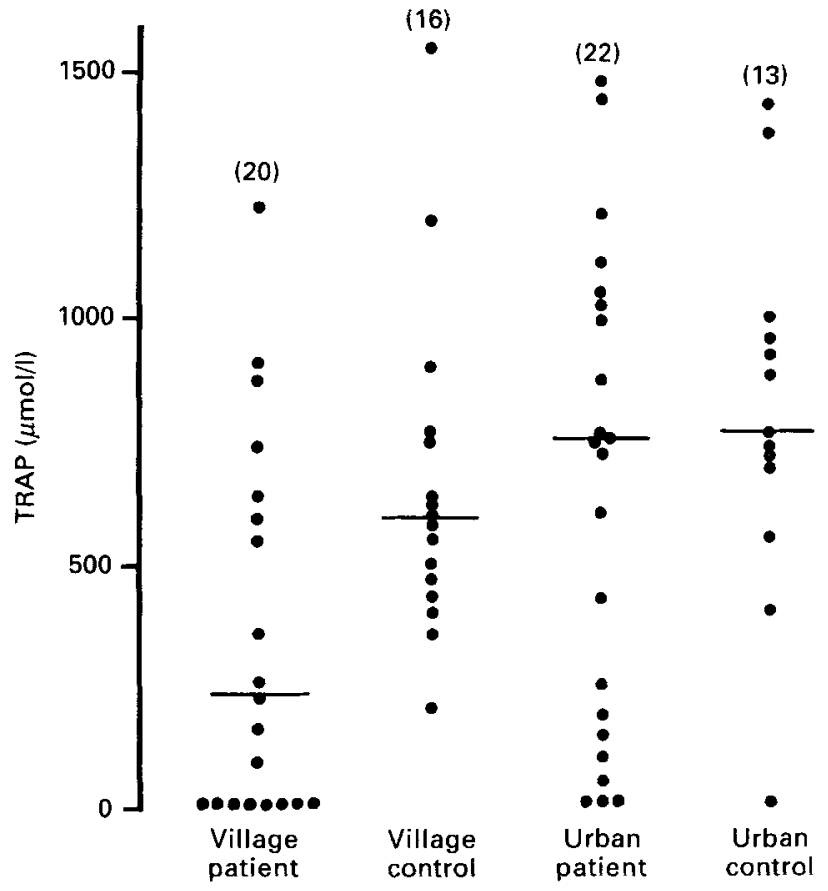

Fig. 1. Distribution of total radical trapping parameter (TRAP) measurements in Thai adults with and without malaria and living in rural and urban districts; no. of subjects in parentheses.

sulphydryl, median values tended to be lowest in the rural malaria patients and slightly higher in the rural controls. Urban malaria patients showed median values very similar to those of the rural controls. The urban controls had the highest median values: significantly different from the two rural groups for all three variables, although only significantly higher than the urban patient group for $\alpha$-tocopherol. There was no difference in proteinsulphydryl value between the two urban groups. The differences in tocopherol values among the groups completely disappeared when $\alpha$-tocopherol was expressed as the tocopherol:cholesterol ratio (Table 1).

MDA was measured in the four groups (Table 2). The highest individual values were obtained in the two groups of malaria patients but the only significant difference between the medians of the groups was between the two control groups. When MDA was expressed relative to cholesterol, however, the median ratio in the village patients was significantly higher than that in either control group. The median ratio in the urban patients was slightly higher than that of urban controls but the difference was not significant. The median concentration of the acute-phase protein, caeruloplasmin, was higher in each group of malaria patients than in their respective control groups. The highest median caeruloplasmin value occurred in the rural malaria patients and the lowest in the urban control group. Median cholesterol values were also lower in the patients than in their respective control groups and lower in the villagers.

At the time of measuring TRAP capacity, ascorbate could not be detected in any of the samples. Plasma extracts prepared from the rural blood samples at the time of collection showed significantly lower ascorbate values in the malaria samples than the controls (Table 3). In addition, when plasma ascorbate concentrations $v$. measured TRAP values of the two 


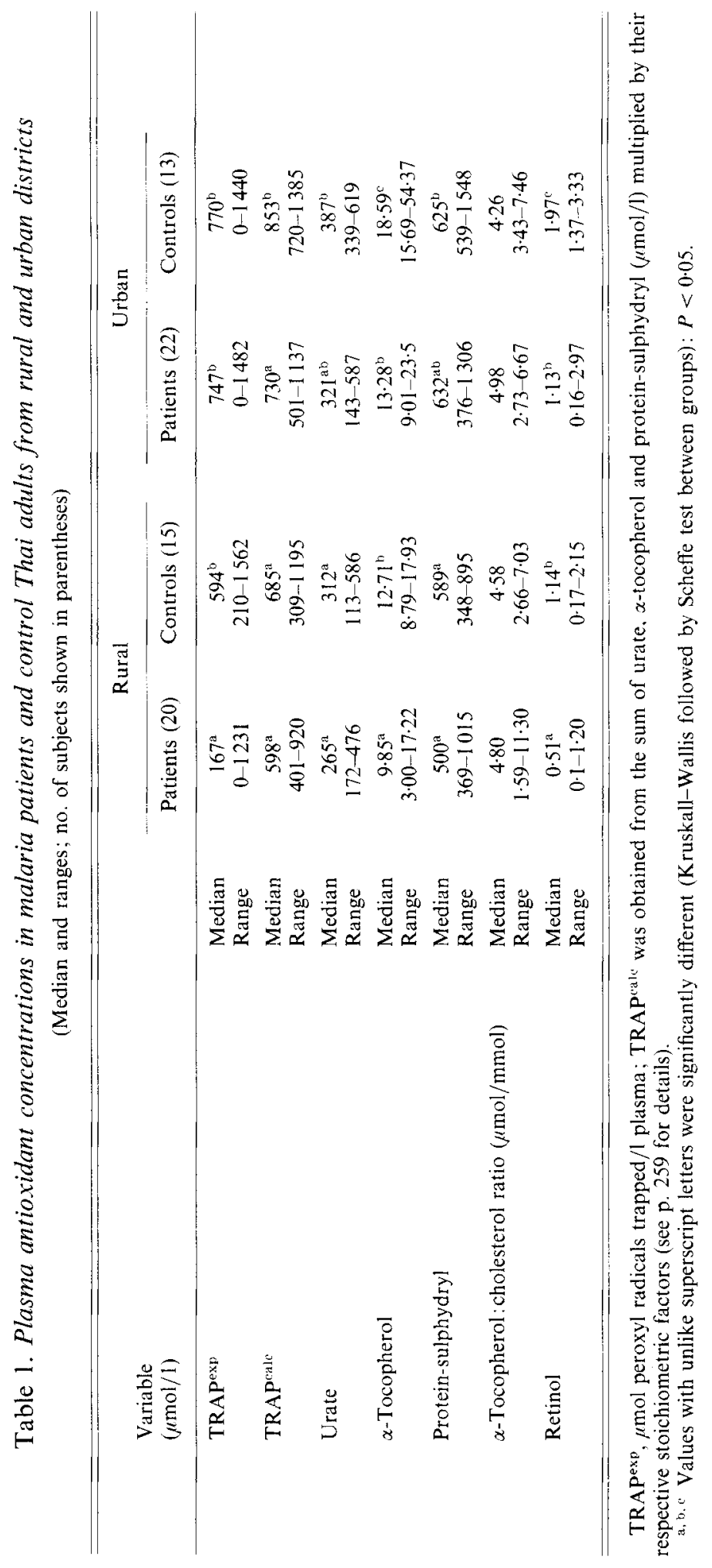




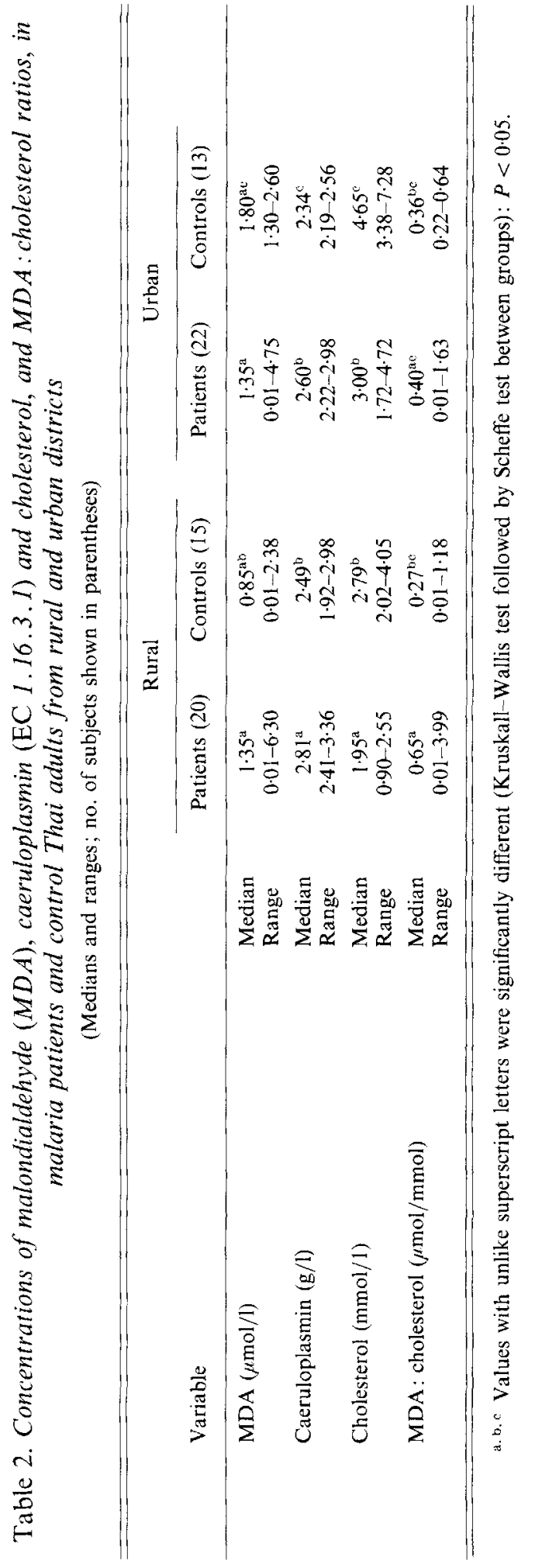


Table 3. Plasma ascorbate ( $\mu$ mol/l) in malaria patients and control Thai adults from a rural district

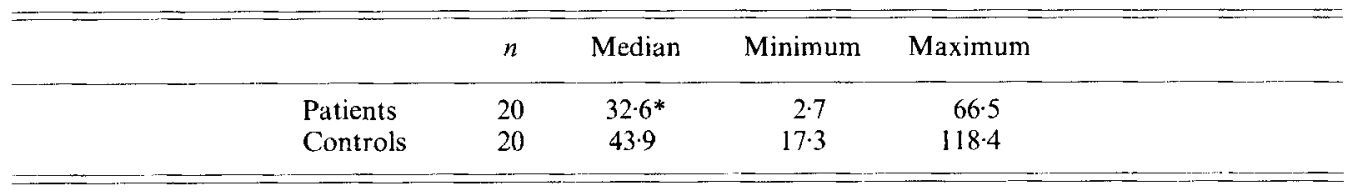

* Median value was significantly different from that for control subjects (Mann-Whitney): $P<0 \cdot 02$.

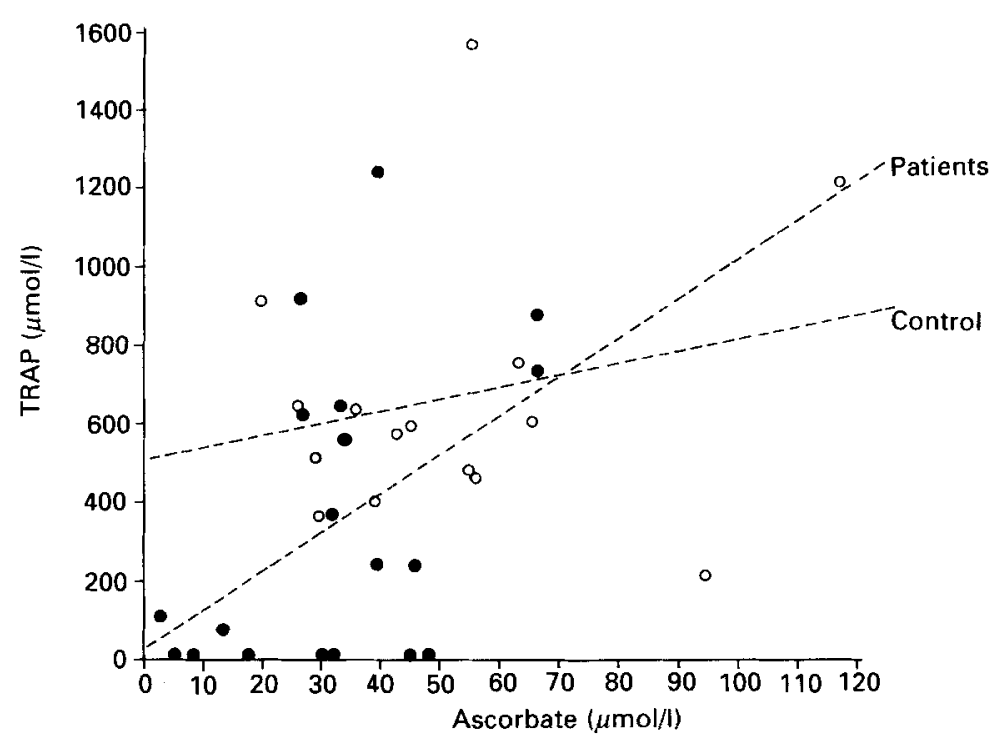

Fig. 2. Relationship between plasma vitamin $C$ concentrations and total radical trapping parameter (TRAP) capacity in malaria patients $(O)$ and control Thai adults $(O)$ from the rural district.

groups of rural inhabitants were plotted, ascorbate was weakly correlated with measured TRAP in the patients $(r 0.449, n 19, P<0.054)$, but there was no indication of any correlation within the control group ( $r$ 0.21, $n$ 15, not significant (Fig. 2).

Plasma retinol (Table 1) and carotenoid concentrations (not shown) were also lower in both groups of malaria patients than their respective controls (R. Singkamani and D. I. Thurnham, unpublished results). Furthermore, plasma retinol concentrations correlated with TRAP values in the malaria patients $(r 0.484, n 43, P<0.001$; Table 4$)$ but not in the control subjects $(r 0 \cdot 295, n 29, P=0 \cdot 12)$. There was a similar relationship between TRAP values and the non-provitamin A carotenoid lutein, but the results did not reach significance (values not shown). Fig. 3 shows the relationship between retinol and TRAP in the two groups of patients separately.

All cases of malaria were mild and were treated in outpatients with antimalarial drugs. At the time of admission to the study, temperature, parasite count and duration of fever were higher in the village than the urban patients but the difference in parasite counts did not reach significance (Table 5). These measurements were compared with TRAP values for the group as a whole and for the village and urban patients separately (Table 4). For the group of patients as a whole there were no significant correlations and, within the individual groups, the only consistent relationship was between TRAP and parasite count 
Table 4. Correlation coefficients for disease indices, malondialdehyde (MDA), $M D A$ : cholesterol and retinol against total radical trapping parameter (TRAP) in malaria petients from Thailand

(No. of subjects in parentheses)

\begin{tabular}{|c|c|c|c|}
\hline Variable & Village & Urban & Combined \\
\hline Temperature $\left(^{\circ}\right)$ & $\begin{array}{c}0.393 \\
(19)\end{array}$ & $\begin{array}{c}-0.379 \dagger \\
(22)\end{array}$ & $\begin{array}{r}-0.223 \\
(41)\end{array}$ \\
\hline Duration of illness (d) & $\begin{array}{c}-0.310 \\
\text { (17) }\end{array}$ & $\begin{array}{l}0.342 \\
(22)\end{array}$ & $\begin{array}{r}-0.077 \\
(39)\end{array}$ \\
\hline Parasite count + & $\begin{array}{r}-0.470 \\
(11)\end{array}$ & $\begin{array}{c}-0.169 \\
(22)\end{array}$ & $\begin{array}{r}-0 \cdot 288 \\
(33)\end{array}$ \\
\hline MDA $(\mu \mathrm{mol} / 1)$ & $\begin{array}{r}-0.387 \\
(19)\end{array}$ & $\begin{array}{c}-0.452^{*} \\
(22)\end{array}$ & $\begin{array}{c}-0 \cdot 419^{* *} \\
(41)\end{array}$ \\
\hline MDA : cholesterol ratio $(\mu \mathrm{mol} / \mathrm{mmol})$ & $\begin{array}{c}-0.385 \\
(19)\end{array}$ & $\begin{array}{c}-0.314 \\
(22)\end{array}$ & $\begin{array}{c}-0 \cdot 384^{* *} \\
(41)\end{array}$ \\
\hline Retinol $(\mu \mathrm{mol} / 1)$ & $\begin{array}{l}0.547^{* *} \\
(20)\end{array}$ & $\begin{array}{l}0.358 \dagger \\
(23)\end{array}$ & $\begin{array}{c}0 \cdot 484^{* * *} \\
(43)\end{array}$ \\
\hline
\end{tabular}

Pearson correlation coefficients were statistically significant: $\dagger P<0 \cdot 1, * P<0.05,{ }^{* *} P<0.02,{ }^{* * *} P<0.001$. $\dagger$ Expressed as $\log _{10}$ parasite counts per 200 white blood cells.

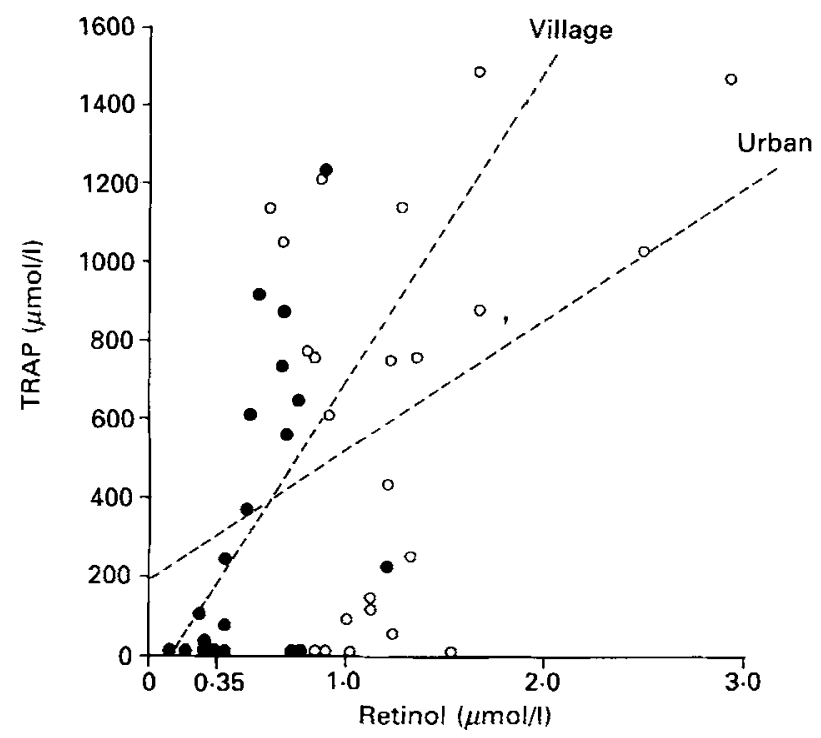

Fig. 3. Relationship between plasma retinol concentrations and total radical trapping parameter (TRAP) measurements in Thai adults with malaria from village $(O)$ and urban $(O)$ communities.

expressed as logarithms to the base ${ }_{10}$ where there were inverse relationships in both groups of patients. MDA was also consistently inversely related to TRAP in combined village and urban patients (Table 4).

Multiple linear-regression analysis was done on the combined patient and control groups separately to determine to what extent the different experimental results explained the variance in TRAP values. Ascorbate was initially not included in this analysis since the 
D. I. THURNHAM AND OTHERS

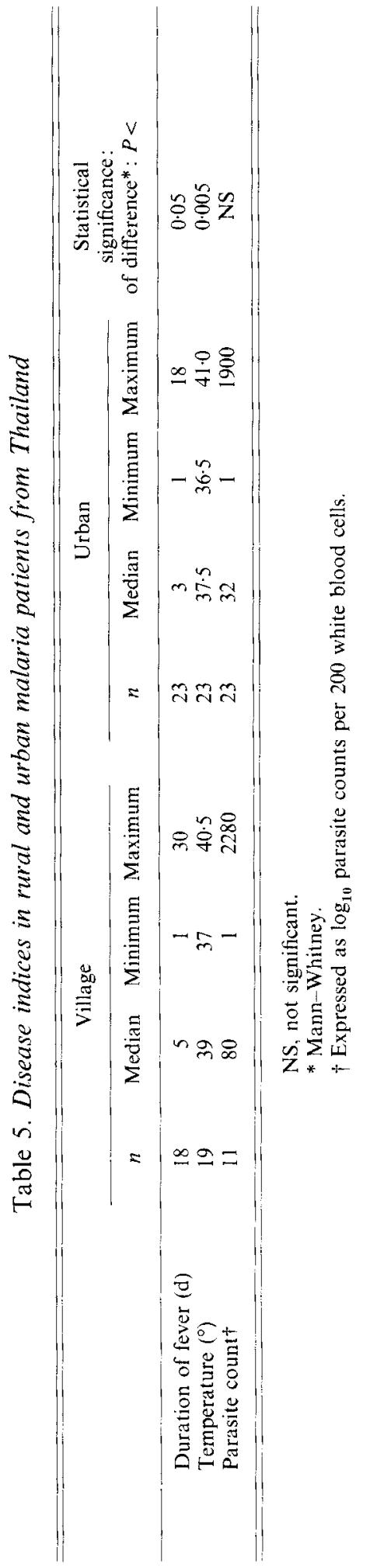


measurements related to the samples at the time of collection and not when TRAP was measured. In both cases, $\alpha$-tocopherol, retinol, individual carotenoids, urate, proteinsulphydryl, caeruloplasmin and MDA were the factors included in the analysis. In the control group, the total variance explained was $31 \%$ and was mostly explained by urate $(25 \%, r 0.5, P<0.02, n 25)$. In the case of the patients, approximately $40 \%$ of the variance in TRAP values could be explained and most of this was attributable to retinol $(25 \%$, $r 0.49, P<0.01, n 40)$ and the remainder to MDA $(18 \%, r-0.42, P<0.01)$. Adding ascorbate into the equation increased the total variance explained to $54 \%$ but the number of subjects was reduced to nineteen. It was previously reported (Thurnham, 1988) that urate followed by ascorbate explained most of the variance in the malaria patients. However, as the earlier analysis was only done on the nineteen rural malaria patients, it may not be as representative as the values reported in the present paper which analysed both patient groups combined.

\section{DISCUSSION}

The TRAP measurements made in the present study confirmed our earlier observations (Thurnham et al. 1988a) that TRAP was too low to measure in a large number of patients with malaria. This was particularly evident for the samples obtained from the rural malaria patients, but even amongst the urban patients almost $25 \%$ had very low TRAP values.

Three of the other measurements made gave values which correlated with TRAP values. These were vitamin $C, M D A$ and retinol. The numbers of patients within the two groups were small and unfortunately we do not have measurements for vitamin $C$ in the urban patients. Furthermore, the relationship between vitamin $C$ and TRAP was weak and may therefore be coincidental, but both MDA and retinol related to TRAP in the two groups of patients in the same manner (Table 4). MDA is a marker of lipid peroxidation (Satoh, 1978 ) and the inverse relationship seen between MDA (or MDA:cholesterol ratios) and TRAP in both groups of patients suggests that the low TRAP values may be linked in some way with the presence of lipid peroxidation in both groups of malaria patients. In a recent study in Indian children, we also found MDA concentrations were higher in those with malaria than in control children (Das et al. 1990). In addition, in the children with malaria, MDA was correlated with riboflavin status, a vitamin that is also believed to exert an antioxidant function by virtue of its coenzyme role in glutathione reductase (NAD(P)H) $(E C 1.6 .4 .2)$ which regenerates reduced glutathione from oxidized glutathione (Das et al. 1988).

The low concentration of vitamin $\mathrm{C}$ in the rural malaria patients may have been due to dietary insufficiency, but plasma vitamin $C$ is often low in infections (Irwin \& Hutchins, 1976) and recent studies in vitro by Frei et al. (1988) suggest that low values may be a direct result of the cellular immune response. These workers showed that in plasma exposed to chemically-stimulated mononuclear phagocytes, vitamin $\mathrm{C}$ was the first antioxidant to be destroyed. Within the body, vitamin $\mathrm{C}$ can in theory be maintained in the reduced form by the shuttle of dehydroascorbate across the erythrocyte membrane for reconversion to ascorbate (Orringer \& Roer, 1979). However, this mechanism may not be able to cope with the level of oxidant stress that occurs in an infection. Alternatively, once blood is removed from the body, oxidant stress may continue but erythrocytes may be depleted of substrate fairly rapidly and be unable to maintain vitamin $C$ in the reduced form. The positive correlation between vitamin $\mathrm{C}$ and TRAP (although no vitamin $\mathrm{C}$ was present in plasma when TRAP was measured) suggests that both may be affected by factors connected to the same oxidant stress. However, in contrast to vitamin $C$, the other three components of TRAP capacity do not appear to deteriorate rapidly on storage. None of the urate values in any group was below the reference range for healthy adults $(120-420 \mu \mathrm{mol} / 1)$. The 
protein content of the serum was not measured but sulphydryl measurements were very similar to those reported by Thurnham et al. (1987) on fresh material and studies on plasma vitamin $\mathrm{E}$ have shown it to be stable for at least 1 year (Thurnham \& Flora, 1988).

The highest individual values for MDA were obtained in the two groups of patients (Table 2). However, the median value in the urban control group appeared greater than that found in the urban malaria patients. This was not significant and a possible explanation can be found if one considers the cholesterol values which were highest in the urban control group. Cholesterol is a general indicator of the level of lipid in the circulation (Thurnham et al. 1986) and cholesterol and polyunsaturated lipid (PUFA) are the main components of the low-density lipoproteins. PUFA is the substrate required for MDA formation and the amount of peroxidized lipid (the precursor of MDA) formed may be related both to the amount of substrate and to the level of peroxidation. That is, the more lipid, the greater the autocatalytic activity, the longer are the potential chains and the greater the amount of MDA formed. If one controls MDA concentrations for the amount of cholesterol in the plasma, then median MDA: cholesterol ratios are higher in the two patient groups, although the difference between the urban patients and controls was not significant (Table 2).

Median caeruloplasmin concentrations were significantly higher in the two groups of patients than their respective controls. Caeruloplasmin is an acute-phase protein, and is believed to function as an antioxidant by converting ferrous-Fe to the ferric form which is then less able to catalyse the Fenton reaction (Gutteridge, 1986). There was no correlation between caeruloplasmin and TRAP concentrations, which probably indicates that the amount of caeruloplasmin was in excess of any free Fe in the malaria plasma and, therefore, not related to any lipid peroxidation taking place. Caeruloplasmin concentrations were highest in the village malaria patients (Table 2). There was no difference between the two groups of patients in parasite count but temperature and the duration of illness were slightly greater in the village than the urban patients (Table 5). Both the higher temperature and a longer duration of illness may have been due to lower resistance because of malnutrition in the villagers, but both could also have been due to greater difficulties in reaching medical facilities in the rural areas. The higher caeruloplasmin concentrations may indicate more severe disease in the rural patients, but also may simply be a reflection of a higher basal caeruloplasmin level pre-infection, as indicated by the level in the village control group.

The higher caeruloplasmin concentrations in the rural than urban control groups may reflect a higher level of morbidity in the rural population. Poor vitamin A status has been associated with increased levels of morbidity (Sommer et al. 1984) and retinol concentrations were lower in both rural groups than the comparable urban groups (Table 1). Retinol was also inversely related to caeruloplasmin in both control subjects $(r-0 \cdot 338$, $P<0 \cdot 1, n 28)$ and patients $(r-0.413, P<0.01, n 43)$. There was also a clear association between retinol and TRAP values, although in the urban patients, this only reached borderline significance (Table 4). Low retinol concentrations in malaria may be linked to the acute-phase response (Thurnham, 1989) as a fall in circulating transthyretin, which binds retinol-binding protein, is an early feature of infection (Smith \& Goodman, 1971; Fleck \& Myers, 1985). An alternative possibility, however, is that retinol is subjected to oxidant stress and destroyed in the environment where other antioxidants are being consumed. As $30 \%$ (six of twenty) of the retinol concentrations in the rural patients were less than $0.35 \mu \mathrm{mol} / 1$, indicating severe vitamin A deficiency, it is very important to decide whether the infection is destroying the vitamin or stimulating a redistribution. These observations do not answer this question but do suggest that more lipid peroxidation is occurring in those situations where low retinol concentrations are found. Carotene 
concentrations showed a similar distribution among the groups to that seen for retinol ( $R$. Singkamani and D. I. Thurnham, unpublished results). However, none of the carotenes measured showed any relationship to TRAP values, but this is probably not surprising as carotenes are not believed to function as antioxidants in the relatively high $\mathrm{O}_{2}$ tensions of the plasma (Burton \& Ingold, 1984).

The lower concentration of retinol in the rural malaria patients than any of the other groups may of course genuinely reflect the poor vitamin A status in this group. Plasma concentrations below $0.35 \mu \mathrm{mol} / 1$ suggest very low liver reserves, which would be associated with clinical signs of deficiency. Recent prospective studies by Indian workers, however, suggest that such low values are the consequence of the infection and not the cause, for similar low retinol concentrations were found in children with measles but these rose to their premeasles level 8 weeks post-infection without any retinol supplementation (Bhaskaram, 1986). Furthermore, studies by Amatayakul et al. (1989) in a similar rural district in Northern Thailand found no evidence of poor liver vitamin A reserves using the relative dose-response test in spite of circulating retinol concentrations similar to those of the rural control group.

Vitamin $C$ was not present in the samples at the time the measurements of TRAP were made. However, other radical-trapping antioxidants were still present in the plasma and measurable by standard methods, as the TRAP ${ }^{\text {calc }}$ measurements indicated (Table 1). The discrepancy between calculated and experimental TRAP values implies that total oxidant stress acting on the malaria plasma is greater than the oxidant stress generated by ABAP. However, as we previously described (Thurnham et al. 1988a), there was again no evidence of any difference in the rate of $\mathrm{O}_{2}$ uptake between cases and controls when all antioxidant had been consumed (values not shown). That is, the apparent free radical generation in the $\mathrm{O}_{2}$ electrode is the same for both patient samples and control samples, and plasma from malaria patients does not appear to be adding to the radicals generated by ABAP. However, we now believe that the apparently low radical-trapping activity measured in malaria plasma is at least partly due to a loss of plasma antioxidants after mixing plasma with the LA and before the sample has been injected into the $\mathrm{O}_{2}$ electrode, due to the presence of a pro-oxidant in malaria plasma (D. I. Thurnham, D. Kwiatkowski, A. V. S. Hill and B. M. Greenwood, unpublished results). This pro-oxidant does not appear to influence the levels of $\alpha$-tocopherol, urate or protein-sulphydryl until the addition in vitro of LA, although the possible effect on vitamin $C$ in vivo or post-phlebotomy cannot be excluded. This recent finding does not detract from the observation that low TRAP values in malaria plasma are associated with markers of lipid peroxidation in the blood of malaria patients.

In conclusion, we have shown that TRAP measurements were lower in village patients with malaria than in those from an urban area, and that low TRAP values were associated with evidence of lipid peroxidation as indicated by raised median concentrations of MDA relative to cholesterol. Low TRAP values were also associated with poor status of vitamins $A$ and C. Caeruloplasmin was highest in the group with low TRAP values. It did not correlate with TRAP but did correlate with retinol in both control subjects and patients. The lowest $\alpha$-tocopherol concentrations were also found in the groups with the lowest TRAP results and may be a consequence of lipid peroxidation, but $\alpha$-tocopherol was not correlated with TRAP and contributed very little in molar terms to the total TRAP capacity. Tocopherol:cholesterol ratios were not different among the groups, and with the exception of a few results in the rural patients suggested that vitamin E status was adequate.

The collection of specimens was carried out with the support of the WHO/World Bank/UNDP Special Programme Research Strengthening Grant. The British Council 
supported R.S. to travel to the UK for the analyses. The authors thank Dr Jedsada Jittapirom, Director of Maejam Hospital, Chiang Mai for excellent cooperation. D.I.T. is grateful for support from the Department of Health, UK.

\section{REFERENCES}

Amatayakul, K., Underwood, B., Ruckphaopunt, S., Singkamani, R., Linpisarn, S., Leelapat, P. \& Thanangkul, O. (1989). Oral contraceptives: effect of long-term use on liver vitamin A storage assessed by the relative dose response test. American Journal of Clinical Nutrition 49, 845-848.

Bhaskaram, P. (1986). Immune response and infection in relation to vitamin A and iron deficiency in children. In Proceedings of XIIth International Congress of Nutrition, pp. 132-135 [T. G. Taylor and N. K. Jenkins, editors]. London: John Libbey.

Blake, D. R., Merry, P., Unsworth, J., Kidd, B. L., Outhwaite, J. M., Ballard, R., Morris, C. J.. Grey, L. \& Lunec, $J$. (1989). Hypoxic-reperfusion injury in the inflamed human joint. Lancet $\mathbf{i}, 289-293$.

Burton, G. W. \& Ingold, K. U. (1984). $\beta$-Carotene: an unusual type of lipid antioxidant. Science 224, 569-573.

Clark, I. A. (1987). Cell-mediated immunity in protection and pathology of malaria. Parasitology Today 3. $300-305$

Clark, I. A., Allison, A. C. \& Cox, F. E. (1976). Protection of mice against Babesia and Plasmodium with BCG. Nature 259, 309-311.

Clark, I. A. \& Hunt, N. H. (1983). Evidence for reactive oxygen intermediates causing haemolysis and parasite death in malaria. Infection and Immunity 39, 1-6.

Clark, I. A., Hunt, N. H. \& Cowden, W. B. (1986). Oxygen-derived free radicals in the pathogenesis of parasitic disease. Advances in Parasitology 25, 1-44.

Das, B. S., Das, D. B., Satpathy, R. N., Patnaik, J. K. \& Bose, T. K. (1988). Riboflavin deficiency and severity of malaria. European Journal of Clinical Nutrition 42, 277-283

Das, B. S., Thurnham, D. 1., Patnaik, J. K., Das, D. B., Satpathy, R. \& Bose, T. K. (1990). Increased plasma lipid peroxidation in riboflavin-deficient, malaria-infected children. American Journal of Clinical Nutrition (In the Press).

Diluzio, N. R. \& Kalish, G. H. (1966). Enhanced peroxidation of lipid in the pathogenesis of acute ethanolinduced liver injury. Gastruenterology 50, 392-396.

Fleck, A. \& Myers, M. A. (1985). Diagnostic and prognostic significance of the acute phase proteins. In The Acute Phase Response to Injury and Infection, pp. 249-271 [A. H. Gordon and A. Koj, editors]. Amsterdam: Elsevier.

Frei, B., Stocker, R. \& Ames, B. N. (1988). Antioxidant defenses and lipid peroxidation in human blood plasma. Proceedings of the National Academy of Sciences, USA 85, 9748-9752.

Gey, K. F. (1986). On the antioxidant hypothesis with regard to arteriosclerosis. Bibliotheca Nutritio Dieta 37, 53-91.

Gutteridge, J. M. C. (1986). Antioxidant properties of the proteins caeruloplasmin, albumin and transferrin. A study of their activity in serum and synovial fluid from patients with rheumatoid arthritis. Biochimica er Biophysica Acta 869, 119-127.

Harman, D. (1984). Free radical theory of ageing: the 'free radical' diseases. Ageing 7, 111-131.

Irwin, M. I. \& Hutchins, B. K. (1976). A conspectus of research on vitamin C requirements of man. Journat of Nutrition 106, 821-879.

Koottathep, S. (1988). Studies on chain-breaking antioxidants. MSc Thesis, University of Birmingham.

Koster, J.F., Biemond, P. \& Swaak, A. J. G. (1986). Intracellular and extracellular sulphydryl levels in rheumatoid arthritis. Annals of Rheumatic Diseases 45, 4446.

Orringer, E. P. \& Roer, M. E. S. (1979). An ascorbate-mediated transmembrane-reducing system of the human erythrocyte. Journal of Clinical Investigation $\mathbf{6 3}, 53-58$.

Satoh, K. (1978). Serum lipid peroxide in cerebrovascular disorders determined by a new colorimetric method. Clinica Chimica Acta 90, 37-43.

Singkamani, R., Kaewichit, R., Wongworapat, K. \& Thurnham, D. I. (1988). Antioxidant capacity in serum of malaria-infected patients. In Proceedings of the XIIh International Congress of Tropical Medicine \& Malaria, International Congress Series 810, p. 256 [P. A. Karger and A. M. Polderman, editors]. Amsterdam: Excerpta Medica.

Slater, T. F. (1984). Free-radical mechanisms in tissue injury. Biochemical Journal 222, 1-15.

Smith, F. R. \& Goodman, D. S. (1971). The effect of diseases of the liver, thyroid, and kidneys on the transport of vitamin A in human plasma. Journal of Clinical Investigation 50, 2426-2436.

Sommer, A., Katz, J. \& Tarwotjo, 1. (1984). Increased risk of respiratory disease and diarrhea in children with preexisting mild vitamin A deficiency. American Joumal of Clinical Nutrition 40, 1090-1095.

Thurnham, D. 1. (1988). Vitamin C (ascorbic acid): antioxidant functions of vitamin $\mathrm{C}$ in disease in man and animals. In Comparative Nutrition, pp. 91-103 [K. Blaxter and I. Macdonald, editors]. London: John Libbey.

Thurnham, D. I. (1989). Vitamin A deficiency and its role in infection. Transactions of the Royal Society of Tropical Medicine 83, 721-723. 
Thurnham, D. I., Davies, J. A., Crump, B. J., Situnayake, R. D. \& Davis, M. (1986). The use of different lipids to express serum tocopherol:lipid ratios for the measurement of vitamin $\mathrm{E}$ status. Annals of Clinical Biochemistry 23, 514-520.

Thurnham, D. I. \& Flora, P. S. (1988). Stability of carotenoids, retinol and tocopherol in stored plasma. Clinical Chemistry 34, 1947.

Thurnham, D. I., Koottathep, S.\& Adelekan, D. A. (1988a). Chain-breaking antioxidants in the blood of malariainfected children. In Free Radicals: Chemistry, Pathology and Medicine, pp. 161-183 [C. Rice-Evans and T. Dormandy, editors]. London: Richelieu Press.

Thurnham, D. I., Situnayake, R. D., Kootathep, B., McConkey, B. \& Davis, M. (1987). Antioxidant status measured by the TRAP assay in rheumatoid arthritis. In Free Radicals, Oxidant Stress and Drug Action, pp. 169-191 [C. Rice-Evans, editor]. London: Richelieu Press.

Thurnham, D. I., Smith, E. \& Flora, P.S. (1988b). Concurrent liquid-chromatographic assay of retinol, $\alpha$-tocopherol, $\beta$-carotene, $\alpha$-carotene, lycopene, and $\beta$-cryptoxanthin in plasma with tocopherol acetate as internal standard. Clinical Chemistry 34, 377--381.

Thurnham, D. I. \& Stephen, J. M. L. (1979). Biochemical methods: Appendix D. In Nutrition and Health in Old Age, Report on Health and Social Subjects No, 16, pp. 191-197. London: H.M. Stationery Office.

Wayner, D. D. M., Burton, G. W., Ingold, K. U., Barclay, L. R. C. \& Locke, S. J. (1987). The relative contributions of vitamin $\mathbf{E}$, urate, ascorbate and proteins to the total peroxyl radical-trapping antioxidant activity of human blood plasma. Biochimica ef Biophysica Acta 92, 408-419.

Wayner, D. D. M., Burton, G. W., Ingold, K. U. \& Locke, S. (1985). Quantitative measurement of the total, peroxyl radical-trapping antioxidant capability of human blood plasma by controlled lipid peroxidation. $F E B S$ Letters 187, 33-37.

Wozencraft, A. O., Dockrell, H. M., Taverne, J., Targett, G. A. T. \& Playfair, J. H. L. (1984). Killing of human malaria parasites by macrophage secretory products. Infection and Immunity 43, 664-669. 